DOI: $10.3901 / J M E .2021 .19 .070$

\title{
基于动作基元的人臂达点运动模式*
}

\author{
赵 京 王承运 张自强 龚世秋 \\ (北京工业大学材料与制造学部 北京 100124)
}

\begin{abstract}
摘要: 为揭示人臂达点运动中人臂末端位置、姿态和自摆角的运动协调关系, 实现机器人的深度仿人运动规划, 对人臂达点 运动的运动模式进行了研究。将人臂动作基元的排列与组合方式定义为人臂达点运动的运动模式, 进行了 3240 次人臂达点 运动实验数据采集与处理, 分析了达点运动阶段和达点区域对运动模式的影响; 提出了基于基元类型、次序变化、运动模式 频率以及基元连接方式频率约束的运动模式相似度指标一一复合约束相似度, 并以此优选出了人臂达点运动的最优运动模式; 基于这一运动模式，利用 7 自由度拟人机械臂复现了人臂的达点运动。
\end{abstract}

关键词: 动作基元; 达点运动; 人机交互; 运动模式相似度；仿人运动

中图分类号: TP242

\section{Motion Pattern of Human Arm Reaching Point Movements Based on the Movement Primitives}

\author{
ZHAO Jing WANG Chengyun ZHANG Ziqiang GONG Shiqiu
}

(Faculty of Materials and Manufacturing, Beijing University of Technology, Beijing 100124)

\begin{abstract}
In order to reveal the coordination relationship between swivel angle, position and posture of the end of the human arm, and to realize the robot's deep human-like motion planning, the motion pattern of the human arm reaching point movement was studied. The arrangement and combination of the movement primitives of the human arm were defined as the motion pattern of the human arm reaching point movement. The experimental data collection and processing of 3240 human arm reaching point were carried out, and the influence of the movement phases and the region of the reaching point on the motion pattern was analyzed. Compound constraint similarity, an index of motion pattern similarity based on constraint of primitive type, order change, frequency of motion pattern and frequency of connection mode of primitive, was proposed, and the optimal motion pattern of human arm reaching point was optimized. Based on this motion pattern, the reaching point movement of the human arm is reproduced by using a 7-DOF anthropomorphic robotic arms.
\end{abstract}

Key words: movement primitives; reaching point movement; human-robot interaction; motion pattern similarity; anthropomorphic movement

\section{0 前言}

人臂达点运动是人类日常生活中最基本的运动 形式, 对其特征的研究不仅可以让人们掌握人臂达 点运动的规律, 还将有助于人们将这些规律应用于 新一代机器人的运动规划中, 从而使人机交互(HRI) 更加自然、便利 ${ }^{[1-2]}$, 进一步提高机器人的人机交互

* 北京市自然科学基金(3192002)和国家自然科学基金(51975008)资助 项目。20201009 收到初稿, 20210124 收到修改稿
能力 ${ }^{[3-4]}$ 。目前国内外学者对于人臂达点运动特征的 研究主要集中在操作空间和关节空间两个方面。

在操作空间中, 人们发现人臂指尖的运动轨迹 大致沿直线, 且速度为钟型分布 ${ }^{[5]}$ 。依据这些特征 设计出两类轨迹生成模型: 基于数据的模型 ${ }^{[6]}$ 和基 于优化的模型 ${ }^{[7-8]}$ 。这类模型的优点是可以生成平滑 的指尖运动轨迹, 但是生成的仿人运动不直观, 且 需要求逆运动学解, 计算量大。在关节空间方面, GIUSEPPE 等 ${ }^{[9]}$ 对日常生活中 30 种达点任务进行了 实验, 拟合出这些达点任务的关节轨迹函数, 通过 
函数主成分分析(FPCA)提取出关节轨迹函数的主 成分, 并以此重新构建各关节运动轨迹。通过减少 函数主成分的数量降低了描述复杂运动和关节运动 规划及控制的难度。但是通用性差, 当出现新的达 点任务时, 需要重新对关节轨迹函数的主成分重新 提取。

此外, 针对关节空间和操作空间提取的运动特 征都不直观问题, 一些学者利用人臂动作基元来表 征人臂达点运动。FANG 等 ${ }^{[10]}$ 根据人的上臂、前臂 和肩膀和手腕之间的虚拟线可组成虚拟三角形, 提 出使用人臂三角形来表示人臂的运动, 并将人臂三 角形应用于拟人臂的仿人运动。GONG 等 ${ }^{[11]}$ 提取出 能够完整表达人臂运动的基本动作基元: 自摆角 $\Phi$ 、 手掌端点(指尖)的位置 $P$ 和方向 $O$ 、操作力 $F$ 。这 四个基本动作基元具有明确物理意义, 易于量化和 识别。

上述这些研究主要集中在人臂达点运动的位移、 速度和加速度规律以及臂姿直观表达的问题上。但 大量的实验结果表明, 人臂达点运动的末端位置、 姿态及自摆角的运动协调关系存在明显的规律性, 定量化地表征这些规律对于进一步提高拟人化程度 具有重要价值。为此, 本文将在文献[11]的基础上, 将动作基元的排列与组合方式定义为运动模式, 通 过研究运动模式揭示人臂运动协调关系。根据人臂 指尖加速度特性将达点运动分为四个连续的运动阶 段, 同时把达点区域按象限分为四个达点区域。基 于大量达点运动实验, 分别提取出达点运动各阶段 各达点区域的运动模式, 提出运动模式的度量指标 一运动模式复合约束相似度, 并以此篮选出能代表 达点区域内运动特性的运动模式。最后, 将人臂达 点运动的运动模式应用于 7 自由度机械臂上进行达 点运动实验, 实现机械臂在达点运动过程中的深度 仿人。

\section{1 人臂动作基元}

\section{1 人臂结构和运动表达}

人的手臂由上臂、前臂和手掌组成, 由于其冗 余结构的存在因此可以灵活地完成复杂的任务。在 生物物理学和机构运动学中, 人的手臂通常被建模 为一个具有七个自由度的串联机构, 肩关节有三个 自由度, 时关节有一个自由度, 腕关节有三个自 由度。

手臂运动的表达对人臂运动的识别和理解至关 重要。人脑对运动的记忆是通过记忆身体的几何特
征和运动轨迹来实现的, 因此引入人臂三角形来丰 富人臂的几何特征, 使人臂运动更加直观 ${ }^{[11]}$ 。人臂 三角形由上臂、前臂和肩膀和手腕之间的虚拟线组 成, 如图 1 所示。它的形状和姿势分别由自摆角和 手腕位置决定, 其中自摆角 $\Phi$ 定义为手臂三角形的 肘部围绕虚线轴 $S W$ 由位置 $E$ 旋转到位置 $E_{1}$ 的旋转 角度 ${ }^{[13]}$ 。

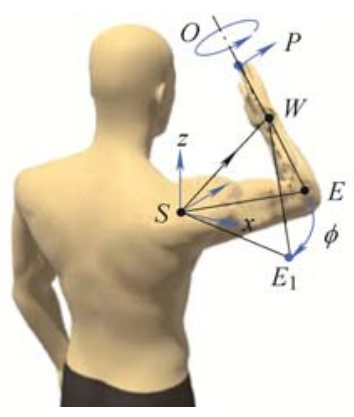

图 1 人臂运动表达 $\left(\triangle S W E\right.$ 和 $\triangle S W E_{1}$ 是人臂三角形, $\Phi$ 是 自摆角, $P$ 和 $O$ 分别是手掌位置和姿态)

然而人的手臂三角形不能表达手掌的运动, 因 此加入了手掌的三种运动信息, 实现了完整的手臂 运动表达, 即手掌端点(指尖)的位置 $(P)$ 和姿态 $(O)$ 以及操作力 $F$ 。其中, $\boldsymbol{P}=[x, y, z], \boldsymbol{O}=\left[r_{x}, r_{y}, r_{z}\right]$ 表 示三个欧拉方向角, $F$ 表示人手或机器人夹持器的 夹持力。

$\Phi, P, O$ 和 $F$ 这四个变量可以充分的表达手臂 的运动。在手臂运动过程中手掌可以当成一个刚体, 那么 $P$ 和 $O$ 可以确定唯一的手腕位置, 而且手腕位 置和自摆角 $\Phi$ 可以确定唯一的人臂三角形形状和姿 态。因此已知的 $\Phi, P$ 和 $O$ 可以确定唯一的手臂构 型, 即由 $\Phi, P$ 和 $O$ 可以得到手臂运动表达式。此 外 $F$ 反映了人手(或机器人夹持器)与外部物体的接 触关系, 这对于判断手或夹持器是否在抓取物体至 关重要。

\section{2 基元定义}

人臂动作基元的提取灵感来自于人类手臂的运 动表达。在一个复杂的手臂运动过程中 $\Phi, P, O$ 和 $F$ 这四个变量很可能会发生变化。单个变量的变化 被视为一个基本运动模块, 因此人臂运动有四个基本 运动模块, 这些基本运动模块定义为人臂基本动作基 元。自摆角旋转角度、指尖位置、指尖方向的变化率 和末端交互力分别用 $\hat{\Phi}(t) 、 \hat{P}(t) 、 \hat{O}(t)$ 和 $F(t)$ 表示。 通过判断它们是否非零, 可以识别出手臂的运动, 例 如, 如果只有 $\hat{\Phi}(t)$ 非零, 这意味着只有自摆角在变 化。将这四个基本动作基元组合在一起, 可以组合成 新的动作基元。通过组合两个不同的基本动作基元 
可以生成 $6\left(C_{4}^{2}=6\right)$ 个新的动作基元; 同样, 通过组 合三个不同的基本动作基元得到 $4\left(C_{4}^{3}=4\right)$ 个新动 作基元; 通过组合所有的基本动作基元生成一个新 动作基元。因此, 通过这样的组合产生了 11 个新的 动作基元。这些新生成的动作基元可以表示为: $\overline{\Phi P}$, $\overline{\Phi O}, \overline{\Phi F}, \overline{P O}, \overline{P F}, \overline{O F}, \overline{\Phi P O}, \overline{\Phi P F}, \overline{\Phi O F}$, $\overline{P O F}, \overline{\Phi P O F}$ ，其中 $\overline{\Phi P}$ 和 $\overline{P \Phi}$ 是等价的。

这些组合基元的运动含义可以通过四种基本基 元的组合含义获得。如 $\overline{\Phi P}$ 的运动含义是关于时间 $\mathrm{t}$ 的函数 $\Phi(t)$ 和 $P(t)$ 运动含义的组合, 即手臂三角 形绕虚拟轴旋转, 同时指尖位置也发生变化。

定义了一个特殊的动作基元 $A(t)$, 这意味着在 $\mathrm{t}$ 时刻没有运动和夹紧力。到目前为止, 已经提出了 16 个动作基元, 把所有的基元放入一个数学集合形 成一个人臂动作基元库(HAMPL), 即

\section{$\{\Phi, P, O, F, \overline{\Phi P}, \overline{\Phi O}, \overline{\Phi F}, \overline{P O}, \overline{P F}, \overline{O F}$, $\overline{\Phi P O}, \overline{\Phi P F}, \overline{\Phi O F}, \overline{P O F}, \overline{\Phi P O F}, A\}$}

人臂动作基元的运动含义可以用一些可以帮助 理解和记忆的图来表示, 如图 2 展示了四个动作基 元图。

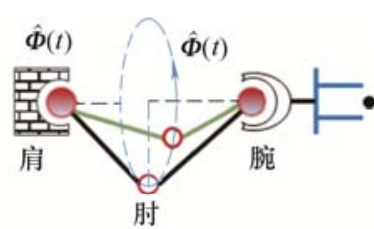

(a) $\bar{\Phi}(t)$

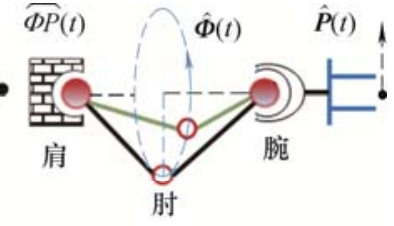

(b) $\overline{\Phi P}(t)$

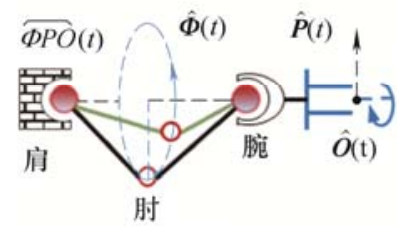

(c) $\overline{\Phi P O}(t)$

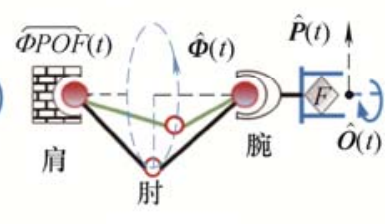

(d) $\overline{\Phi P O F}(t)$
图 2 动作基元图

\section{2 人臂达点运动}

\section{1 运动模式的提取}

为了反映达点运动中人臂末端位置、姿态和自 摆角的运动协调关系, 把动作基元的排列与组合方 式定义为运动模式。通过研究从达点运动中提取的 运动模式揭示人臂运动协调关系。

判断基本动作基元 $\hat{\Phi}(t) 、 \hat{P}(t)$ 和 $\hat{O}(t)$ 是否为零 可以识别出手臂的运动, 因而对达点运动中基本动 作基元变化率是否为零进行识别, 统计出每帧中基 元变化率不为零的基元类型。达点运动每帧中基元
变化率不为零的基元类型有 $10\left(C_{3}^{1}+C_{3}^{2}+C_{3}^{3}=10\right)$ 种, 如 $\overline{\Phi P O} 、 \overline{\Phi P}$ 等。任取一次达点运动实验数据, 对每帧中基元变化率不为零的基元类型按时间顺序 进行排列为

$$
\underbrace{\overline{\Phi P O} \ldots \overline{\Phi P O}}_{k} \underbrace{\overline{\Phi P} \ldots \overline{\Phi P}}_{m} \underbrace{\overline{\Phi P O} \ldots \overline{\Phi P O}}_{n}
$$

因为前 $k$ 帧的基元类型都为 $\overline{\Phi P O}$, 所以直接 用 $\Phi P O$ 表示前 $k$ 帧的基元类型, 其他帧基元类型也 按此法进行表示。根据运动模式的定义, 此次达点 运动的运动模式可表示为 $\Phi$ PO- $Ф$ P-ФPO, 这里 “-” 表示基元类型的前后连接。按照上述方式对所有实 验数据的运动模式进行统计。

\section{2 达点运动实验}

文献[14]证明了分析人类手臂的结构和运动可 以有效地促进机器人在日常生活中的实际应用。我 们期望从人类手臂运动中提取出人臂运动模式的规 律, 并将这些规律应用于拟人化手臂的运动规划中。 这些提取的规律可以帮助人类更好地理解和规划拟 人化手臂的动作, 也可以使机器人的动作变得像人 一样。为此进行了人臂达点运动捕捉实验, 对人臂 达点运动模式的规律进行了初步探索。根据人臂运 动模式的定义, 提取出所有运动样本中的运动模式。

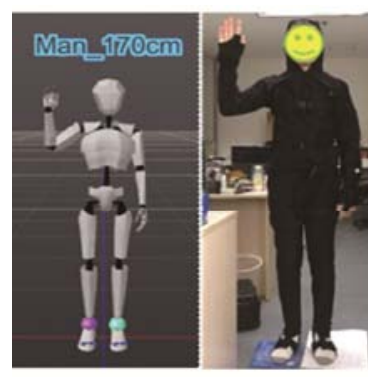

(a)

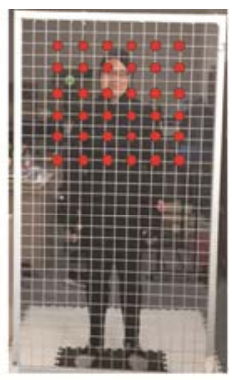

(b)
图 3 实验设备及达点实验

人臂达点运动捕捉实验的实验设备如图 $3 \mathrm{a}$ 所 示, 实验场景如图 3b 所示, 图 3b 中相邻点的间距 均为 $10 \mathrm{~cm}$, 共有 36 个点组成达点工作空间。在实 验过程中，每个参与者分别站在达点工作空间前的 $30 \mathrm{~cm} 、 40 \mathrm{~cm} 、 50 \mathrm{~cm}$ 处, 达点空间的中心大致与 参与者的肩部对齐。达点运动开始前右臂处于自然 下垂状态，每个参与者被要求以日常生活中的速度 和舒适的节奏来完成达点运动, 达点结束时右手指 尖指向图 $3 b$ 中的标记点。共有 10 名参与者进行达 点实验, 每个标记点重复三次, 总共完成 3240 次实 验。OptiTrack 运动捕捉系统(图 3a)以 $120 \mathrm{~Hz}$ 的速 率记录参与者的达点运动, 采集每个参与者达点运 动中手臂各关节角度值, 最后通过关节角和基元 
之间的映射关系, 计算出基本动作基元 $\Phi 、 P 、 O$ 的值。

\section{3 达点运动模式分析}

对人臂达点运动数据进行分析发现按照运动模 式定义统计常见运动模式有: $\Phi P O 、 \Phi P O-\Phi P O-\Phi P$ 、 ФРО-ФР 、 ФР-ФРО-ФР-ФРО-ФР 、 ФР-ФРО-РО$\Phi P O-\Phi P$ 等。这种方式统计的运动模式不便于进行 精细分析, 为了使运动模式更精确应用于机器人仿 人运动, 对达点运动的运动模式进行分阶段和达点 区域分析。

位置是加速度对时间的两次积分, 直接控制加 速度可以对轨迹做更精确快速的跟踪, 因此根据人 臂末端加速度特征将三维空间中人臂达点运动分解 为四个连续的运动阶段: 起始阶段、中间阶段 1 、 中间阶段 2、结束阶段。起始阶段为加速度由 0 增 加到最大值时期间运动; 中间阶段 1 为加速度由最 大值减小至 0 时期间运动; 中间阶段 2 为加速度由 0 减小至最小值时期间运动; 结束阶段为加速度由 最小值增加至 0 时期间运动。

将达点区域各点的运动模式进行统计发现运动 模式在不同的象限具有明显的差异, 由此把达点运 动中达点的象限位置作为影响运动模式的外部 因素。

\section{1 运动模式与运动阶段的关系}

对达点运动各个运动阶段出现频率较高的前两 种运动模式进行统计, 如图 4 所示。

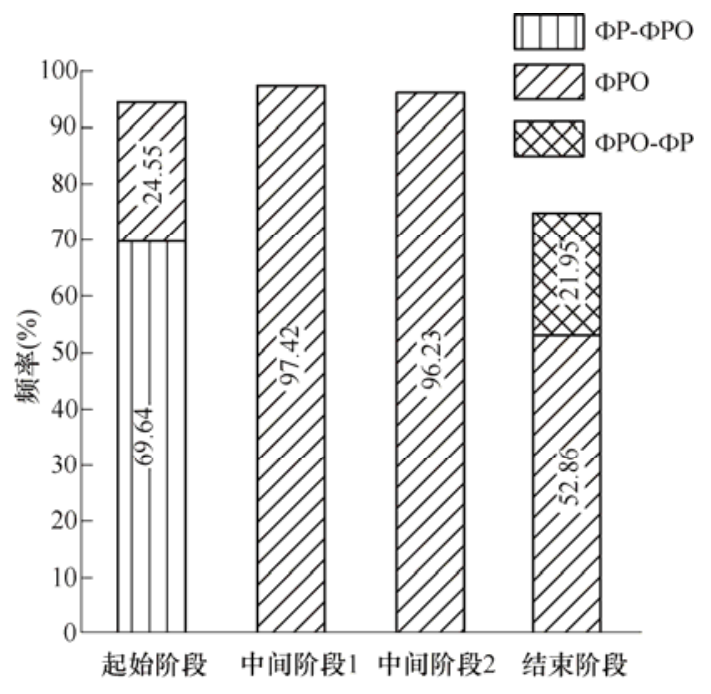

图 4 四个阶段运动模式频率统计

由图 4 可知起始阶段、中间阶段 1 和中间阶段 2 这三个运动阶段的前两种运动模式频率之和达 $90 \%$, 其中中间阶段 1 和中间阶段 2 运动模式主要
为 $\Phi P O$, 出现频率达到 $98 \%$, 说明达点运动的中间 阶段受外界影响小。结束阶段前两种运动模式频率 之和达 70\%, 其他运动模式频率不到 30\%, 显示出 结束阶段运动模式多样化。

运动模式与运动阶段的关系可用于机器人对人 臂达点运动的预测, 当检测到人臂运动模式为 ФP-ФPO 时, 可推测出人臂运动处于起始阶段; 当 检测到人臂运动模式为 $\Phi P O-\Phi P$ 时, 表示人臂达点 运动完成; 当检测到运动模式为 $\Phi P O$ 时, 表示人臂 达点运动处于达点运动的中间阶段, 正进行快速达 点运动。通过检测人臂的运动模式预测人臂的运动 阶段, 提前对机器人的运动进行规划。

\section{2 运动模式与达点区域关系}

把每个阶段运动模式出现频率最高的两种运动 模式按达点位置进行统计, 如图 5 所示。
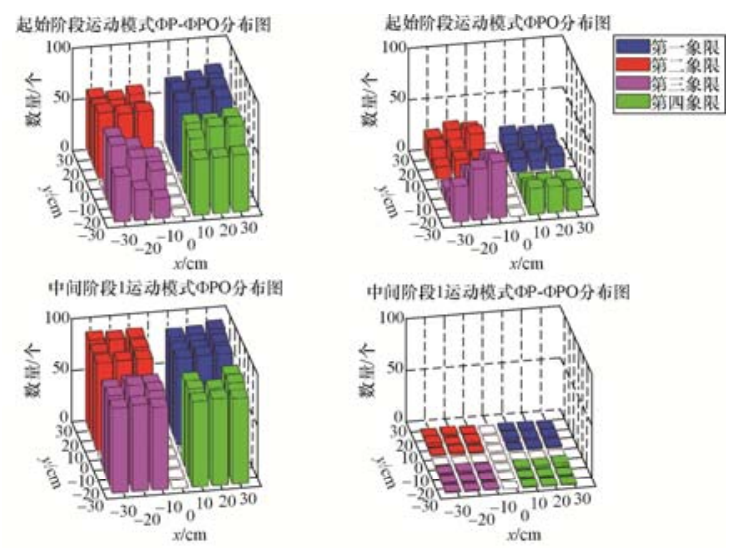

(a)
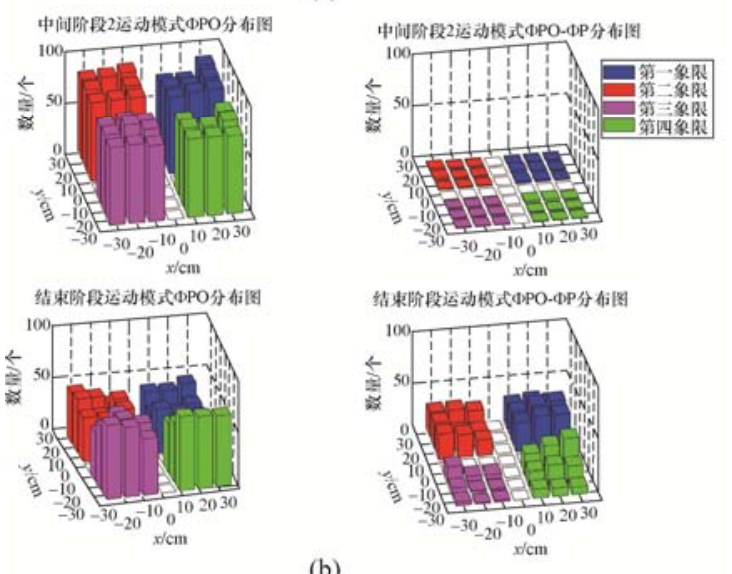

(b)

图 5 达点运动模式分布

从图 5 中可看出中间阶段 1 和中间阶段 2 运动 模式在整个达点区域分布较均匀, 说明在达点运动 中的中间阶段 1 和中间阶段 2 中运动模式与达点位 置无关, 拟人臂在进行仿人运动时运动的中间阶段 直接使用 $\Phi P O$ 运动模式。

起始阶段运动模式 $\Phi P-\Phi P O$ 在达点各点处分布 都较多, 但在第三象限与其他象限比分布相对较少, 
说明在人臂达点运动中起始阶段在第一、二、四象 限更倾向于使用 $\Phi \mathrm{P}-\Phi P O$ 运动模式, 在第三象限倾 向于交替使用 $\Phi P O$ 和 $\Phi P-\Phi P O$ 运动模式。

结束阶段运动模式 $\Phi P O$ 和 $\Phi P O-\Phi P$ 在第一象 限分布较均匀, 两种运动模式在第一象限都有相当 数量的分布; 在第三象限 $\Phi P O$ 运动模式明显比 $\Phi P O-\Phi P$ 运动模式出现频率大; 在第二、四象限 $\Phi P O$ 运动模式出现频率大于 $\Phi P O-\Phi P$ 运动模式出现频率, 但是频率相差不太大。 象限分布较少, 但 $\Phi P O$ 运动模式在三、四象限分布 并没有显著增加, 而是有其他运动模式如 $\Phi P O-\Phi P-\Phi P O$ 等, 以上分析说明人臂达点运动结束 阶段运动模式可选择性较多。

\section{3 运动模式及基元连接频率}

经分析基元连接频率越高, 运动模式中含有该 连接基元的概率越大, 即基元连接频率与运动模式 频率成正相关关系。为下节研究运动模式的度量, 本节对运动模式和基元连接频率进行分析统计。由 以上分析可知人臂达点运动的中间阶段 1 和中间阶 段 2 运动模式比较集中, 大概率使用 $\Phi P O$ 运动模式, 而起始阶段和结束阶段运动模式较分散, 特别是结 束阶段运动模式, 由此对结束阶段的运动模式进行 频率统计, 统计出结束阶段运动模式的频率如表 1 所示, 其他运动阶段处理方式和结束阶段一样。

表 1 结束阶段运动模式频率统计

\begin{tabular}{|c|c|c|c|}
\hline $\begin{array}{l}\text { 结束阶段第一 } \\
\text { 象限运动模式 }\end{array}$ & 频率(\%) & $\begin{array}{l}\text { 结束阶段第二 } \\
\text { 象限运动模式 }\end{array}$ & $\begin{array}{r}\begin{array}{r}\text { 频率 } \\
(\%)\end{array} \\
\end{array}$ \\
\hline ФРО & 42.62 & ФРО & 54.39 \\
\hline ФРО-ФР & 38.34 & ФРО-ФР & 29.04 \\
\hline ФРО-ФР-ФРО & 6.99 & ФРО-РО-ФРО & 6.52 \\
\hline ФРО-ФР-ФРО-ФР & 6.09 & ФРО-ФР-ФРО & 3.97 \\
\hline ФР & 4.40 & ФРО-ФР-ФРО-ФР & 3.68 \\
\hline ФРО-ФР-ФРО-ФР-ФРО & 1.55 & ФРО-РО & 2.41 \\
\hline $\begin{array}{l}\text { 结束阶段第三 } \\
\text { 象限运动模式 }\end{array}$ & 频率(\%) & $\begin{array}{l}\text { 结束阶段第四 } \\
\text { 象限运动模式 }\end{array}$ & $\begin{array}{c}\text { 频率 } \\
(\%)\end{array}$ \\
\hline ФРО & 71.96 & ФРО & 64.38 \\
\hline ФРО-ФР & 10.26 & ФРО-ФР & 22.69 \\
\hline ФРО-РО-ФРО & 6.02 & ФРО-ФР-ФРО & 4.75 \\
\hline ФРО-ФР-ФРО & 6.02 & ФРО-ФР-ФРО-ФР & 3.96 \\
\hline ФРО-ФР-ФРО-ФР & 3.42 & $\Phi \mathrm{P}$ & 2.51 \\
\hline ФРО-РО & 2.33 & ФРО-РО-ФРО & 1.72 \\
\hline
\end{tabular}

结束阶段运动模式中共出现 $\Phi P O 、 \Phi P 、 P O$ 和 $P$ 四种基元, 这四种基元之间进行两两组合或单个
基元定义为基元的连接方式，如 $\Phi P О-、 \Phi P O-\Phi P 、$ $\mathrm{P}$-等。基元连接方式共有 16 种 $\left(C_{4}^{1}+C_{4}^{2}=16\right)$, 由表 1 知在结束阶段运动模式中共出现 7 种连接方式分 别为: $\Phi P O-、 \Phi P O-\Phi P 、 \Phi P O-P O 、 \Phi P-、 \Phi P-\Phi P O 、$ PO-和 PO- $\Phi$ PO, 其他连接方式出现频率全为零。

根据基元连接方式定义，基元连接方式的频率 计算公式为

$$
P L_{j}=\left(\sum_{i}^{n_{t o}} N_{i j} * N P_{i}\right) /\left(\sum_{i}^{n} N E_{i} * N P_{i}\right)
$$

式中, $P L_{j}$ 为第 $j$ 种连接方式的频率; $n_{t o}$ 为运动模式 数量; $N_{i j}$ 为第 $i$ 种运动模式中出现第 $j$ 种连接方式 的次数; $N P_{i}$ 为第 $i$ 种运动模式出现的次数; $N E_{i}$ 为 第 $i$ 种运动模式连接方式的个数。

按式(1)计算出结束阶段四个象限基元连接方 式的频率如表 2 所示。

表 2 结束阶段四个象限基元连接方式频率统计 $(\%)$

\begin{tabular}{ccccc}
\hline 连接方式 & 第一象限 & 第二象限 & 第三象限 & 第四象限 \\
\hline ФРО- & 28.93 & 39.68 & 57.17 & 48.03 \\
$\Phi P О-\Phi Р ~$ & 34.28 & 24.70 & 15.74 & 23.97 \\
$\Phi P O-P O$ & 0 & 5.46 & 5.68 & 1.16 \\
ФР- & 27.62 & 20.02 & 9.31 & 19.77 \\
ФР-ФРО & 9.13 & 4.68 & 6.42 & 5.90 \\
РО- & 0 & 1.47 & 1.58 & 0 \\
РО-ФРО & 0 & 3.99 & 4.10 & 1.16 \\
\hline
\end{tabular}

\section{4 达点运动模式的确定}

由第 3 节可知, 达点运动模式与达点运动阶 段和达点位置有一定关系, 即不同的运动阶段运 动模式不同, 不同的达点位置具有不同的运动模 式，因而分阶段和达点区域确定运动模式。每个 达点区域又有多种运动模式, 需要从多种运动模 式中选取最优运动模式来代表该达点区域的运动 模式。优选运动模式时应考虑运动模式内部因素 和外部因素的影响, 因而选取的最优运动模式应 该满足如下条件。

(1) 选取的运动模式尽可能真实的反应人臂达 点运动特性, 尽可能结合所有运动模式的特点。

(2) 选取的运动模式应尽可能体现出运动模式 及基元连接方式出现频率的影响, 即选取的运动模 式倾向于出现频率高的运动模式和含有高频率基元 连接方式的运动模式, 消除外界因素对优选运动模 式的影响。

针对选取运动模式的两个条件, 本节提出运动 
模式的度量方式一运动模式复合约束相似度, 通过 计算运动模式相似度优选运动模式。

\section{1 运动模式的度量}

一般来说运动模式的度量可以从频率角度 出发, 出现频率最高的运动模式作为该阶段最 优运动模式。但是这种运动模式优选方式有如 下问题。

(1) 这种度量方式不能说明优选的运动模式能 真实的体现人臂达点运动特性, 即通过高频率优选 运动模式的方式未能结合所有运动模式本身的特点。 如表 1 中结束阶段第二象限运动模式, $\Phi P O$ 出现的 频率为 $54.39 \%$, 其他运动模式出现频率为 $45.61 \%$, 从频率角度优选的运动模式为 $\Phi P O$, 舍弃了 $45.61 \%$ 运动模式所具有的特性, 因而从频率角度优选的方 式未能体现 $\Phi P O$ 结合所有运动模式的 特点。

(2) 出现多种运动模式频率相差不大时无法确 定最优运动模式。如表 1 中人臂达点运动结束阶段 第一象限运动模式中 $\Phi P O$ 运动模式出现次数为 329 次, 频率为 $42.62 \%$, $\Phi P O-\Phi P$ 运动模式出现次数为 296 次, 频率为 $38.34 \%$ 。由于人臂达点运动中受各 种因数的影响, 如设备精度、个人习惯、个人的预 判能力等都能影响运动模式出现的次数, 因而当多 种运动模式频率相当时无法确定最优运动模式。

\subsection{1 运动模式相似度}

由运动模式的定义可知运动模式由不同的基元 类型按照一定次序组合而成如 $\Phi$ Р- $Ф P O 、 \Phi P O-\Phi P 、$ $\Phi P O-\Phi P-\Phi P O$ 等, 从中可看出基元类型及次序体现 出每个运动模式的不同, 不同运动模式基元类型及 次序变化程度越小则运动模式相似程度越高。受字 符串相似度定义启发 ${ }^{[15]}$, 提出利用运动模式相似度 衡量运动模式的相似程度。通过度量运动模式的相 似度优选运动模式。

类比字符串相似度计算方式, 设两个运动模式 中一个为原运动模式 $s$, 另一个为目标运动模式 $t$, $s$ 由 $m$ 个基元组成, $t$ 由 $n$ 个基元组成, 定义两个运 动模式的相似度为 $\operatorname{sim}(t, s)$, 两个运动模式的相似 度计算公式为

$$
\operatorname{sim}(t, s)=\frac{n+m-d(t, s)}{n+m}
$$

式中, $d(t, s)$ 为两个运动模式中基元类型和次序变 化次数, 即原运动模式通过改变基元的类型和次序 达到和目标运动模式基元类型和次序相同需要改变 的次数, 改变一次的成本为 1 , 即难度系数为 1 , 改 变总次数可由 Levenshtein Distance 算法 ${ }^{[15]}$ 计算最小 改变次数。
运动模式相似度不仅考虑了运动模式包含的基 元类型, 还考虑了基元次序因素(内部因素), 即选 取最优运动模式时考虑了所有运动模式的特点, 但 是未体现运动模式和基元连接方式频率因素(外部 因素)对运动模式优选的影响。

\subsection{2 运动模式复合约束相似度}

针对运动模式相似度未考虑运动模式和基元连 接方式出现频率对运动模式相似程度影响的缺点, 提出复合约束相似度。每种运动模式中含有一种及 以上连接方式, 每种连接方式出现的频率不同。为 体现连接方式对运动模式相似度的影响, 采用连接 方式的频率衡量基元类型及次序被改变的成本大小， 连接方式出现频率越高, 对应基元类型及次序被改 变成本越大。如结束阶段第一象限基元连接方式 ФPO- $\Phi \mathrm{P}$ 出现频率为 34.28\%, 改变 $\Phi \mathrm{PO}-\Phi \mathrm{P}$ 连接方 式的成本为 0.3428 。同理, 目标运动模式频率越高, 原运动模式被改变的成本越小。

根据如上分析结合运动模式相似度计算公式, 得到在连接方式和运动模式频率复合约束下的运动 模式复合约束相似度计算公式

$$
\operatorname{simc}(t, s)=\left(m+n-\left(\sum_{i}^{n l_{t}} P_{L}\right)\left(1-P_{t}\right)\right) /(m+n)
$$

式中, $\operatorname{simc}(t, s)$ 为复合约束相似度, $n l_{t}$ 为原运动模 式变为目标运动模式基元变化总次数, $P_{L}$ 为连接方 式频率, $m 、 n$ 分别为原运动模式和目标运动模式包 含的基元个数, $P_{t}$ 为目标运动模式的频率。

利用式(3)计算运动阶段中运动模式两两间相 似度

$$
\operatorname{Simc}(t, s)=\left[\begin{array}{cccc}
\operatorname{simc}\left(t_{1}, s_{1}\right) & \operatorname{simc}\left(t_{1}, s_{2}\right) & \cdots & \operatorname{simc}\left(t_{1}, s_{\mathrm{K}}\right) \\
\operatorname{simc}\left(t_{2}, s_{1}\right) & \operatorname{simc}\left(t_{2}, s_{2}\right) & \cdots & \operatorname{simc}\left(t_{2}, s_{\mathrm{K}}\right) \\
\vdots & \vdots & & \vdots \\
\operatorname{simc}\left(t_{\mathrm{L}}, s_{1}\right) & \operatorname{simc}\left(t_{\mathrm{L}}, s_{2}\right) & \cdots & \operatorname{simc}\left(t_{\mathrm{L}}, s_{\mathrm{K}}\right)
\end{array}\right] \text { (4) }
$$

式中, $K$ 为原运动模式的数量, $L$ 为目标运动模式 的数量。为测量目标运动模式和所有运动模式的相 似程度, 计算目标运动模式和所有原运动模式复合 约束相似度的平均值

$$
\overline{\operatorname{Simc}}\left(t_{j}, s\right)=\sum_{i}^{K} \operatorname{Simc}(j, i) / K
$$

式中, $\overline{\operatorname{Simc}}\left(t_{j}, s\right)$ 为目标运动模式 $t_{j}$ 和所有原运动模 式复合约束相似度的平均值。

\section{2 运动模式笁选}

本节对结束阶段第一、二、三、四象限的运动 模式利用复合相似度进行运动模式篮选，其他阶段 
按同样的方式进行运动模式篮选。结束阶段四个象 限的运动模式如表 1 所示, 按式(5)分别计算结束阶 段四个象限运动模式的复合相似度平均值, 结果如 图 6 所示。

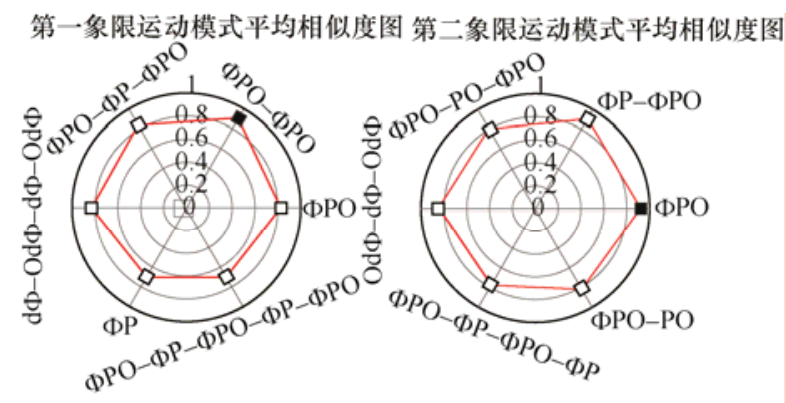

第二象限运动模式平均相似度图 第四象限运动模式平均相似度图

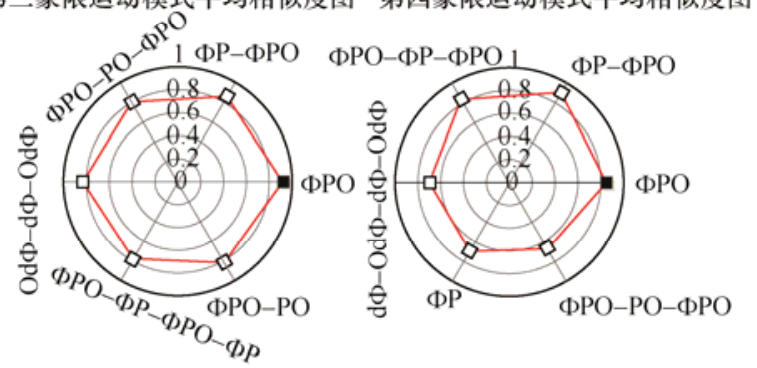

复合约束运动模式 - 最优运动模式 平均相似度

图 6 运动模式平均相似度图

由表 1 可知人臂达点运动的结束阶段第一象 限运动模式 $\Phi P O$ 和 $\Phi P O-\Phi P$ 频率相当, ФPO-ФP 频率略大于 $\Phi P O$ 频率, 由图 6 可知在复合约束下, 由运动模式相似度䇥选出的最优运动模式为 $\Phi P O-\Phi P$, 即 $\Phi P O-\Phi P$ 与其他运动模式在复合约 束条件下相似程度最高, $\Phi P O-\Phi P$ 体现了所有运 动模式的特点。

表 1 显示结束阶段第二、三象限出现的运动模 式相同频率不等, 在通过运动模式复合约束相似度 笁选后, 优选的运动模式为 $\Phi P O$ (图 6)。由此可知 运动模式复合约束相似度优选运动模式时, 在结合 所有运动模式特点的情况下倾向于优选频率高的运 动模式。

由以上分析可看出运动模式复合约束相似度很 好的结合了基元类型和次序、运动模式频率和基元 连接方式频率之间的联系。有效的结合了所有运动 模式的特点, 同时消除了外界因素对优选运动模式 的影响。

为使运动模式能够应用于机器人仿人运动规 划, 利用运动模式复合约束相似度优选出人臂 达点运动各阶段各个象限最优运动模式, 如表 3 所示。

\begin{tabular}{|c|c|c|}
\hline \multicolumn{2}{|c|}{ 象限与阶段 } & 复合约束 \\
\hline \multirow{4}{*}{ 第一象限 } & 初始阶段 & ФР-ФРО \\
\hline & 中间阶段 1 & ФРО \\
\hline & 中间阶段 2 & ФРО \\
\hline & 结束阶段 & ФРО-ФР \\
\hline \multirow{4}{*}{ 第二象限 } & 初始阶段 & ФР-ФРО \\
\hline & 中间阶段 1 & ФРО \\
\hline & 中间阶段 2 & ФРО \\
\hline & 结束阶段 & ФРО \\
\hline \multirow{4}{*}{ 第三象限 } & 初始阶段 & ФР-ФРО \\
\hline & 中间阶段 1 & ФРО \\
\hline & 中间阶段 2 & ФРО \\
\hline & 结束阶段 & ФРО \\
\hline \multirow{4}{*}{ 第四象限 } & 初始阶段 & ФР-ФРО \\
\hline & 中间阶段 1 & ФРО \\
\hline & 中间阶段 2 & ФРО \\
\hline & 结束阶段 & ФРО \\
\hline
\end{tabular}

\section{5 应用实例}

下面取第二象限的运动模式应用于 7 自由度 iiwa 机械臂上进行达点运动实验。在第二象限中 达点运动的起始阶段运动模式为 $\Phi P-\Phi P O$, 中间 阶段 1 、中间阶段 2 和结束阶段的运动模式都为 $\Phi P O$, 因此整个运动阶段运动模式简化为 ФР-ФРО。

由[11]可知人臂达点运动基元 $\Phi 、 P 、 O$ 运动规 律可用如下公式表示

$$
\begin{aligned}
& \phi(t)=\phi_{0}+\int_{0}^{t} \hat{\boldsymbol{\Phi}}(t) \mathrm{d} t \\
& \boldsymbol{P}(t)=\boldsymbol{P}_{0}+\int_{0}^{t} \hat{\boldsymbol{P}}(t) \mathrm{d} t \\
& \boldsymbol{O}(t)=\boldsymbol{O}_{0}+\int_{0}^{t} \hat{\boldsymbol{O}}(t) \mathrm{d} t
\end{aligned}
$$

式中, $\hat{\boldsymbol{\Phi}}(t)=0.5 B(1-\cos (2 \pi t / T)), \phi_{0}$ 为自摆角初 始值, $\hat{\boldsymbol{P}}(t)=\left(\boldsymbol{P}_{0}-\boldsymbol{P}_{f}\right)\left(60 \tau^{3}-30 \tau^{4}-30 \tau^{2}\right) / t_{f}, \boldsymbol{P}_{0}$ 和 $\boldsymbol{P}_{f}$ 分别为末端初始位置和结束位置, $\hat{\boldsymbol{O}}(t)=0.5 A(1-\cos (2 \pi / T)), \boldsymbol{O}_{0}$ 为末端姿态初始值, 参数详细信息见文献[11]。

在运动模式应用实例中各基元的初始值和结束 值分别为

$$
\begin{gathered}
\phi_{0}=-\pi / 3, \phi_{f}=-\pi / 2 \\
\boldsymbol{P}_{0}=[0,500,50], \boldsymbol{P}_{f}=[-400,400,600]
\end{gathered}
$$


$\boldsymbol{O}_{0}=[-\pi / 3, \pi / 10,-\pi / 15], \boldsymbol{O}_{f}=[\pi / 10, \pi / 3, \pi / 3]$

$\phi_{0} 、 \phi_{f} 、 \boldsymbol{O}_{0}$ 和 $\boldsymbol{O}_{f}$ 的单位为 $\mathrm{rad}, \boldsymbol{P}_{0}$ 和 $\boldsymbol{P}_{f}$ 的单位为 $\mathrm{mm}$ 。

根据式(6)、(7)、(8)和各基元的初始值以及结束 值对基元运动轨迹进行规划, 并通过基元运动轨迹 和基元与关节角之间的映射关系求解各关节轨 迹 ${ }^{[13]}$ 。

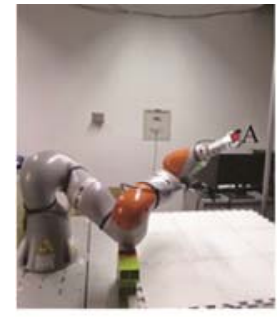

(a)

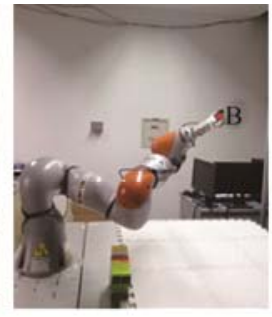

(b)

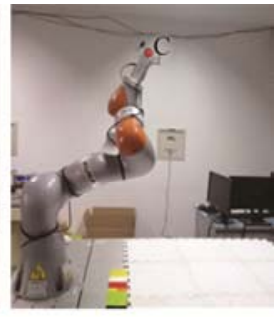

(c)
图 7 机械臂达点运动实验

图 7 为机械臂应用 $\Phi P-\Phi P O$ 运动模式由 $\mathrm{A}$ 点到 $\mathrm{C}$ 点的达点运动实验图。图 7a 机械臂达点运动的起 始位姿, 图 $7 \mathrm{~b}$ 为达点过程中机械臂的末端姿态 $(O)$ 开始发生变化时的位姿, 图 7c 为达点运动结束时机 械臂的位姿。

通过比较图 7 中机械臂位姿变化可知机械臂在 完成达点运动时使用了运动模式 $\Phi P-\Phi P O$ 。对比图 $7 \mathrm{a} 、 7 \mathrm{~b}$ 中机械臂的位姿可知机械臂自摆角和末端位 置发生了改变, 但末端姿态保持不变, 即机械臂由 图 7a 位姿到图 $7 \mathrm{~b}$ 位姿需要通过控制基元 $\Phi$ 和 $P$ 的 运动来实现。对比图 7b、7c 中机械臂的位姿可知机 械臂自摆角、末端姿态和位置都发生了改变, 即机 械臂由图 $7 \mathrm{~b}$ 位姿到图 7c 位姿需要通过控制基元 $\Phi$ 、 $\mathrm{P}$ 和 $\mathrm{O}$ 的运动来实现, 因此机械臂整个达点运动阶 段的运动模式为 $\Phi P-\Phi P O$ 。通过机械臂达点运动实 例证明了人臂运动模式可以应用于机械臂运动规划, 以此来提高机械臂自摆角、末端位置和姿态的运动 协调性, 从而实现机械臂的深度仿人运动规划。

\section{6 结论}

利用人臂动作基元的排列与组合方式定义了人 臂达点运动的运动模式, 通过对运动模式的研究, 揭示了人臂达点运动过程中人臂末端位置、姿态和 自摆角的运动协调关系, 深化了人们对人臂达点运 动规律的认知, 为机器人的深度仿人运动规划奠定 了基础。

(1) 不同运动阶段、不同达点区域运动模式不 同。达点运动的起始阶段出现频率最高的运动模式
为 $\Phi \mathrm{P}-\Phi \mathrm{PO}(70 \%)$, 中间阶段出现频率最高的运动模 式为 $\Phi P O(98 \%)$, 结束阶段出现频率最高的为 $\Phi P O(52 \%)$ 。达点运动的起始阶段和结束阶段运动模 式种类较多, 分布较分散, 与达点位置有关, 中间 阶段运动模式比较集中并且与达点区域相关性较低, 受外部因素影响小。不同阶段运动模式的差异性可 用于人臂运动预测, 方便人机交互中机器人根据人 臂运动来规划自身运动, 提高了人机交互效率。

(2) 复合约束的运动模式相似度结合了内部因 素(动作基元类型和次序)和外部因素(因实验设备、 个人习惯等引起的运动模式及基元连接方式频率变 化)对运动模式的影响, 针对达点区域内出现多种运 动模式且出现频率相差不大时, 基于复合约束的运 动模式相似度可以篮选出能代表达点区域内所有运 动模式特性的最优运动模式。

（3）机械臂达点运动实验证明了体现运动协调性 的运动模式可以应用于拟人臂运动规划, 使拟人臂的 动作深度仿人。

\section{参 考 文 献}

[1] YANG P, FENG G, CHENKUN Q I, et al. Human-tracking strategies for a six-legged rescue robot based on distance and view[J]. Chinese Journal of Mechanical Engineering, 2016, 29(002): 219-230.

[2] 王梅, 卢熙昌. 基于人体动作姿态识别的机器人仿人运 动[J]. 机械工程学报, 2016，52(21): 26-34.

WANG Mei, LU Xichang. Humanoid motion of manipulator that based on human-posture recognition[J]. Journal of Mechanical Engineering, 2016, 52(21): 26-34.

[3] PARK J S, PARK C, MANOCHA D. I-Planner : intention-aware motion planning using learning-based human motion prediction[J]. The International Journal of Robotics Research, 2019, 38(1): 23-39.

[4] CHANG J, LI B, WANG C, et al. Evaluation method on steering for the shape-shifting robot in different configurations $[\mathrm{J}]$. Chinese Journal of Mechanical Engineering, 2016, 29(1): 21-32.

[5] MORASSO P. Spatial control of arm movements[J]. Experimental Brain Research, 1981, 42(1): 223-227.

[6] ZANCHETTIN A M, BASCETTA L, ROCCO P. Achieving humanlike motion: Resolving redundancy for anthropomorphic industrial manipulators[J]. IEEE Robotics \& Automation Magazine, 2013, 20(4): 131-138.

[7] FLUSH T. The coordination of arm movements : an experimentally confirmed mathematical model[J]. J. 
Neuroences, 1985, 5(7): 1688-1703.

[8] UNO Y, KAWATO M, SUZUKI R. Formation and control of optimal trajectory in human multijoint arm movement[J]. Biological Cybernetics, 1989, 61(2): 89-101.

[9] AVERTA G, SANTINA C D, VALENZA G, et al. Exploiting upper-limb functional principal components for human-like motion generation of anthropomorphic robots[J]. Journal of Neuro Engineering and Rehabilitation, 2020, 17(1): 40-55.

[10] FANG Cheng, DING Xinglun, et al. A^2ML: A general human-inspired motion language for anthropomorphic arms based on movement primitives[J]. Robotics and Autonomous Systems, 2018, 111(10): 60-88.

[11] GONG Shiqiu, ZHAO Jing, ZHANG Ziqiang, et al. Task motion planning for anthropomorphic arms based on human arm movement primitives[J]. Industrial Robot, 2020, 47(5): 669-681.

[12] DING Xinglun, FANG Cheng. A novel method of motion planning for an anthropomorphic arm based on movement primitives[J]. Mechatronics, IEEE/ASME Transactions on, 2013, 18(2): 624-636.

[13] 赵京, 龚世秋, 张自强. 7 自由度拟人臂仿人运动的逆
运动学解析解 [J]. 机械工程学报, 2018, 54(21): 25-32. ZHAO Jing, GONG Shiqiu, ZHANG Ziqiang. Analytical inverse kinematics of anthropomorphic movements for 7-DOF humanoid manipulators[J]. Journal of Mechanical Engineering, 2018, 54(21): 25-32.

[14] KÉVIN S, SABOURIN L, STEPHAN F, et al. Analysis of the human arm gesture for optimizing cutting process in ham deboning with a redundant robotic cell[J]. Industrial Robot, 2014, 41(2): 1-8.

[15] ZHANG Shengnan, HU Yan, BIAN Guangrong. Research on string similarity algorithm based on levenshtein distance[C]// 2017 IEEE 2nd Advanced Information Technology, Electronic and Automation Control Conference (IAEAC), Chongqing China: IEEE, 2017: 2247-2251

作者简介: 赵京, 男, 1961 年出生, 博士, 教授, 博士研究生导师。主要 研究方向为机器人运动学和动力学。

E-mail: zhaojing@bjut.edu.cn

王承运, 男, 1992 年出生, 博士研究生。主要研究方向为机器人仿人运动。 E-mail: 623935104@qq.com

张自强(通信作者), 男, 1987 年出生, 博士, 副教授, 硕士研究生导师。主 要研究方向为仿生机器人技术。

E-mail: zzq06520011@163.com

龚世秋, 男, 1992 年出生, 博士研究生。主要研究方向为机器人仿人运动。 E-mail: gsq01041@163.com 